\title{
Clinical evaluation of plasma exchange and high dose intravenous immunoglobulin in a patient with Isaacs' syndrome
}

\author{
Akiko Ishii, Akito Hayashi, Norio Ohkoshi, Eiichi Oguni, Mutsuko Maeda, Yuji Ueda, \\ Kazuhiro Ishii, Keisuke Arasaki, Hidehiro Mizusawa, Shin'ichi Shoji
}

\begin{abstract}
The clinical effects of plasma exchange and high dose intravenous immunoglobulin (IVIg) were evaluated in a 41 year old woman with Isaacs' syndrome. After double filtration plasma exchange, symptoms almost disappeared for two to three weeks and the recorded continuous muscle action potentials were considerably decreased. Symptoms recurred within a few months. On the other hand, IVIg worsened the symptoms of the disorder: during and after IVIg at a dose of $0.2 \mathrm{~g} / \mathrm{kg} / \mathrm{day}$ (total $50 \mathrm{~g}$ ), widespread myokymia, pseudomyotonia, and muscle cramps gradually increased. Symptoms improved after another course of plasma exchange.
\end{abstract}

$(\mathcal{F}$ Neurol Neurosurg Psychiatry 1994;57:840-842)

Isaacs' syndrome is a rare disorder characterised by muscle stiffness, impaired relaxation after contraction (pseudomyotonia), and generalised myokymia. ${ }^{2}$ The cause of the disorder is still obscure, but previous reports have suggested that an immune mechanism may be involved. ${ }^{3-6}$ Plasma exchange has reduced pseudomyotonia in the disorder. ${ }^{56}$ High dose intravenous immunoglobulin (IVIg) has been used successfully to treat Guillain-Barré syndrome ${ }^{7}$ and other presumably immunological disorders affecting peripheral nerve ${ }^{8}$ or muscle. ${ }^{9}$ We evaluated the effects of plasma exchange and IVIg in a patient with Isaacs' syndrome who showed an insufficient response to pharmacological treatment.

\section{Case report}

A 34 year old woman developed symptoms of twitching in her calf muscles and difficulty in releasing her hand grip in 1985. She first came under a doctor's care in 1987 and peripheral nerve block and regional infusion of curare halted the spontaneous muscle activity, but she continued to have persistent muscle activity at rest and during sleep. Therefore, Isaacs' syndrome was diagnosed. She was treated with phenytoin (325 mg daily) and carbamazepine ( $800 \mathrm{mg}$ daily) at a hospital in the United States for a year, which reduced symptoms to some extent. Her symptoms then gradually worsened even though she was taking phenytoin and carbamazepine. She was admitted to our hospital in July 1992. There was no family history of neuromuscular disease. She had been treated with a bronchodilator for a condition diagnosed as asthma at the age of 38 years. Physical examination revealed no abnormalities (including asthma) other than hyperhidrosis. Neurological examination showed that her mental state, cranial nerves, and sensory and cerebellar functions were normal. Hand grip release was slow (pseudomyotonia). No percussion myotonia was present. Her calves were very enlarged. Myokymia occurred in almost all leg and hand muscles and there was severe involvement of the gastrocnemius muscle, which resembled a bag of worms. Her ankles were occasionally fixed in the plantar flexion position due to muscle cramping. Muscle tone was increased in the lower extremities. Tendon reflexes were present, except at the ankles. The Chvostek sign was negative. Laboratory tests revealed an increase in serum $\gamma$-glutamyl transpeptidase and cholinesterase activity due to mild fatty liver and the presence of mild anaemia due to iron deficiency. All other tests, including urinalysis, immunoglobulin levels, cerebrospinal fluid analysis, and thyroid function tests, were normal. No autoantibodies were detected.

An EMG study with a concentric needle electrode demonstrated spontaneous motor unit potentials with various firing patterns, including high frequency bursts and multiple and irregular discharges. Motor and sensory nerve conduction velocities were within normal ranges. The time from maximal voluntary contraction of muscles to electrical silence was measured as EMG relaxation time. The duration of the EMG relaxation time after five second maximal voluntary contraction of the gastrocnemius muscle was over five minutes. Duration of the relaxation time after three second maximal voluntary contraction of the abductor pollicis brevis was about one minute.

Plasma exchange and IVIg were evaluated in terms of their effects on clinical symptoms and EMG findings. The patient's medications were not changed during the treatment. 
Informed consent concerning all examinations and treatments was obtained from the patient beforehand.

Double filtration plasma exchange was performed with PVA (Plasmacure, Kuraray, Japan) as the first filter and Evaflux 2A (Kuraray, Japan) as the second. Plasma exchange was carried out three times on an alternate day schedule. The total amount of plasma processed was 5.58 litres. Symptoms improved two days after completing the third session of plasma exchange. The degree and frequency of resting myokymia apparently decreased. She was unable to release her hand grip before the last session, but two days after the session the grip release time was 3.3 seconds, which further decreased to $1 \cdot 3$ seconds after seven days.

Two weeks after plasma exchange, the duration of the EMG relaxation time after gastrocnemius muscle five second maximal voluntary contraction and abductor pollicis brevis three second maximal voluntary contraction shortened considerably (fig 1). Symptoms gradually worsened over the next few weeks. One month after plasma exchange, continuous muscle activity reappeared and the duration of EMG relaxation time after gastrocnemius muscle five second maximal voluntary contraction and abductor pollicis brevis three second maximal voluntary contraction increased (fig 2). Muscle symptoms deteriorated to pretreatment levels about two months after double filtration plasma exchange.

About five months after double filtration plasma exchange, immunoglobulin (Venoglobin-I, Midorijyuji, Japan) was given intravenously at a dose of $0.2 \mathrm{~g} / \mathrm{kg}$ per day (total $50 \mathrm{~g}$ ) for five consecutive days. The patient experienced general fatigue two days after the start of IVIg. Dysphagia increased on day three and dyspnoea appeared on day 4. An EMG study showed an increase in continu-

At rest

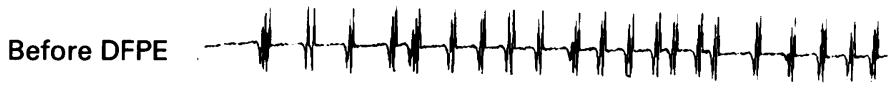

After DFPE $\underset{100 \mathrm{~ms}}{\mathrm{~J}} 200 \mu \mathrm{V}$

Before DFPE

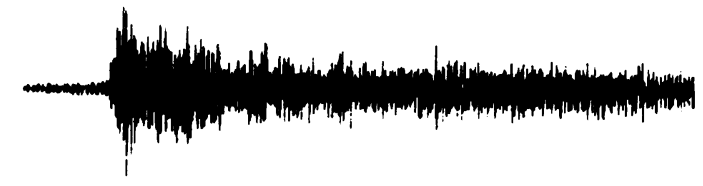

After DFPE

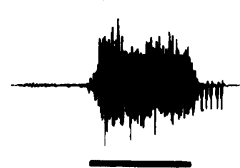

Figure 1 Spontaneous continuous motor action potentials (two upper traces) and the EMG relaxation time after abductor pollicis brevis three second maximal voluntary contraction (two lower traces) were recorded before and after double filtration plasma exchange (DFPE). The solid line indicates the duration of maximal voluntary contraction of the muscle.

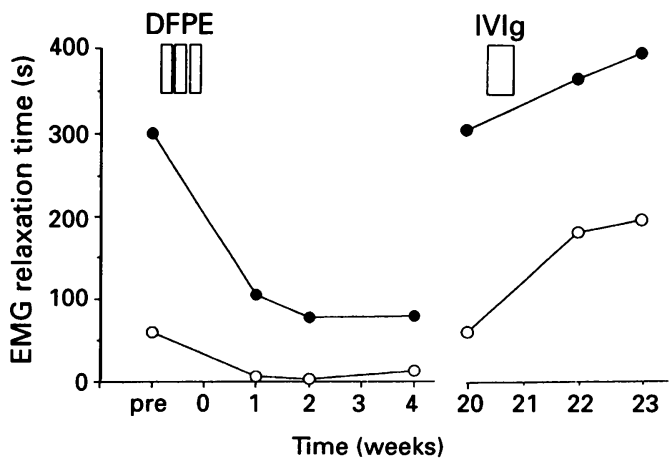

Figure 2 The duration of the EMG relaxation time after gastrocnemius muscle five second maximal voluntary contraction (closed circles) and after abductor pollicis brevis three second maximal voluntary contraction (open circles) during and after double filtration plasma exchange (DFPE) and IVIg. Each block on the left side represents a session of DFPE on an alternate day schedule. The block on the right side represents a five day course of IVIg.

ous muscle activity. The duration of EMG relaxation time was more than five minutes after gastrocnemius muscle five second maximal voluntary contraction and more than one minute after abductor pollicis brevis three second maximal voluntary contraction, similar to pretreatment values (fig 2). During two weeks after IVIg, muscle cramps gradually increased. Symptoms improved after another course of double diffusion plasma exchange.

\section{Discussion}

Continuous muscle action potentials, a typical pathological feature in Isaacs' syndrome, ${ }^{12}$ are manifest clinically by myokymia and pseudomyotonia. The pathogenic mechanism of pseudomyotonia is not known. Previous reports have suggested that Isaacs' syndrome may be an autoimmune disorder. Immune complexes have been detected in patients with the disorder. ${ }^{34}$ When Sinha et al ${ }^{5}$ treated a case of Isaacs' syndrome with plasma exchange, symptoms almost disappeared and suggested the presence of some pathogenic humoral factors in the plasma of Isaacs' syndrome in terms of vitro studies. Moreover, Newsom-Davis et al described the effects of this treatment on four patients with neuromyotonia ${ }^{6}$ The effect of plasma exchange with a double filtration method in our patient as well as previous cases ${ }^{56}$ was confirmed and is consistent with Isaacs' syndrome when it has an autoimmune aetiology.

IVIg has been used successfully to treat Guillain-Barré syndrome ${ }^{7}$ and other presumably immunological disorders affecting peripheral nerve ${ }^{8}$ and muscle. ${ }^{9}$ The mechanism is unclear, but potential mechanisms include Fc receptor blockade or solubilisation of immune complexes by immunoglobulin. ${ }^{10-12}$ Therefore, IVIg would be expected to be useful in Isaacs' syndrome, which may be related to an immune mechanism based on the effect of plasma exchange. Our patient developed widespread myokymia, pseudomyotonia, muscle cramps, dysphagia, and dyspnoea, however, during and after receiving IVIg. The dysphagia and dyspnoea were 
probably the result of laryngeal involvement. Jackson et al ${ }^{13}$ described a case of Isaacs' syndrome with laryngeal involvement that had been diagnosed as asthma. Our study showed that IVIg worsened the symptoms of Isaacs' syndrome. The reason is unclear. Recently, two groups reported that IVIg worsened the symptoms of patients with Guillain-Barré, ${ }^{14-16}$ and IVIg may have a similar adverse effect in Isaacs' syndrome.

In conclusion, plasma exchange was confirmed as a useful treatment for Isaacs' syndrome, suggesting that an immune mechanism may be involved in the disorder. On the other hand, our report is the first study of the use of IVIg in Isaacs' syndrome and further trials of IVIg are needed before the usefulness of this treatment can be assessed.

1 Isaacs $\mathrm{H}$. A syndrome of continuous muscle-fibre activity. f Neurol Neurosurg Psychiatry 1961;24:319-25.

2 Isaacs $H$. Continuous muscle fibre activity in an Indian male with additional evidence of terminal motor fibre abnormality. $\mathcal{f}$ Neurol Neurosurg Psychiatry 1967;30: 126-33.

3 Nagashima $\mathrm{T}$, Kamegai $\mathrm{M}$, Hirose $\mathrm{K}$, et al. Creatine kinase (CK)-linked IgA in Isaacs' syndrome. An immune complex disease? f Neurol Sci 1985;67:269-76.

4 Kuwasaki N, Shoji H, Tominaga $H$, Kaji M, Nonaka $K$. A case of Isaacs' syndrome-Continuous muscle fiber activity syndrome. Brain Nerve 1986;38:531-6.

5 Sinha S, Newsom-Davis J, Mills K, Byrne N, Lang B Vincent A. Autoimmune aetiology for acquired neuromyotonia (Isaacs' syndrome). Lancet 1991;338:75-7.

6 Newsom-Davis J, Mills KR. Immunological associations of acquired neuromyotonia (Isaacs' syndrome): Report of five cases and literature review. Brain 1993;116 453-69.

7 Kleyweg RP, van der Meché FGA, Meulstee J. Treatment of Guillain-Barré syndrome with high-dose gamma globulin. Neurology 1988;38:1639-41.

8 Nobile-Orazio E, Meucci N, Barbieri S, Carpo M, Scarlato G. High-dose intravenous immunoglobulin therapy in multifocal motor neuropathy. Neurology therapy in multifo

9 Soueidan SA, Dalakas MC. Treatment of inclusion-body myositis with high-dose intravenous immunoglobulin. Neurology 1993;43:876-9.

10 Delfraissy JF, Tehernia G, Laurian Y, Wallon C, Gallanand $\mathrm{P}$, Dormont J. Suppressor cell function after intravenous gamma globulin treatment in adult chronic idiopathic thrombocytopenic purpura. $\mathrm{Br} \mathcal{f}$ Haematol 1985;60:315-22.

11 Salama A, Mueller-Eckhardt C, Kiefel V. Effect of intravenous immunoglobulin in immune thrombocytopenia. Lancet 1983;ii: 193-5.

12 Sultan Y, Kazatchkine MD, Maisonneuve P, Nydegger UE. Antiidiotypic suppression of autoantibodies to factor VIII (antihaemophilic factor) by high-dose intravenous gammaglobulin. Lancet 1984;ii:765-8.

13 Jackson DL, Satya-Murti S, Davis L, Drachman DB Isaacs syndrome with laryngeal involvement: An unusual presentation of myokymia. Neurology 1979;29: unusual

14 Bleck TP. IVIg for GBS: Potential problems in the alphabet soup. Neurology 1993;43:857-8.

15 Irani DN, Cornblath DR, Chaudhry V, Borel C, Hanley DF. Relapse in Guillain-Barré syndrome after treatmen with human immune globulin. Neurology 1993;43: 872-5.

16 Castro LHM, Ropper AH. Human globulin infusion in Guillain-Barré syndrome: Worsening during and after treatment. Neurology 1993;43:1034-6. 